\title{
Elaboración de un modelo de evaluación estadístico, para reducir las tasas de accidentabilidad en la Mina Uchucchacua
}

\author{
Development of a statistical evaluation model, to reduce accident rates at the \\ Uchucchacua Mine
}

Carlos Enrique Rodríguez Vigo ${ }^{1}$

Recibido: Enero 2020 - Aprobado: Junio 2020 - Publicado: Junio 2020

\begin{abstract}
RESUMEN
Esta investigación permitió evaluar los resultados de la gestión de riesgos en una operación minera. Se destaca la importancia de adoptar medidas preventivas para controlar las causas de la accidentabilidad. Con tal fin, se desarrolla una Metodología que permite identificar y dar la máxima atención a los riesgos críticos existentes, determinados a partir de evaluar y analizar la información que brindan los Informes de Investigación de Accidentes. Así, se reconocen las características del trabajo predominantes en la ocurrencia de los accidentes, para atenderlas sistemáticamente y evitar los daños que generan. Esta metodología utiliza el Principio de Pareto, para encontrar de manera práctica, aquellas pocas características del trabajo que aún influyen en la accidentabilidad, en las que debemos enfocar prioritariamente la gestión. También permite identificar el 'público objetivo' que es el más afectado por los daños ocurridos. Toda esta información adecuadamente manejada puede facilitar el actuar preventivamente para controlar los riesgos en el trabajo, buscando proteger al personal y evitar nuevos o eventos repetitivos que los afecten.
\end{abstract}

Palabras claves: Metodología; riesgos críticos; Principio de Pareto; accidentabilidad; público objetivo; prevención de accidentes.

\begin{abstract}
This investigation allowed to evaluate the results of risk management in a mining operation. This highlights the importance of adopting preventive measures to control the causes of accidents. To this end, a methodology is developed that allows identifying and giving maximum attention to the existing critical risks, determined from evaluating and analyzing the information provided in the Accident Investigation Reports. Thus, the predominant work characteristics are recognized in the occurrence of accidents, to systematically address them and avoid the damage they generate. This methodology uses the Pareto Principle, to find in a practical way, those few characteristics of the work that still influence the accident rate, in which we must focus our management as a priority. It also allows identifying the 'target audience' that is the most affected by the damage. All this properly managed information can facilitate acting preventively to control risks at work, seeking to protect staff and avoid new or repetitive events that affect them.
\end{abstract}

Keywords: Methodology; critical risks; Pareto principle; accident rate; target audience; objective public; accident prevention.

\footnotetext{
${ }^{1}$ Docente de Facultad de Ingeniería Geológica, Minera, Metalúrgica y Geográfica, Universidad Nacional Mayor de San Marcos. Lima, Perú.

E-mail: crodriguezv1@unmsm.edu.pe
} 


\section{INTRODUCCIÓN}

La accidentabilidad en la actividad minera es preocupación en diferentes latitudes, no solo por su impacto económico, sino por la necesidad de preservar el recurso humano (Guzmán, L., s. f.), (Van Neck, 2012).

En el Perú, según el («Decreto Supremo D.S. $N^{\circ}$ 024-2016-EM», 2016), accidente laboral es definido como "todo suceso repentino que sobrevenga por causa o con ocasión del trabajo y que produzca en el trabajador una lesión orgánica, una perturbación funcional, una invalidez o la muerte".

Para (Velásquez Palacios et al., 2016) “existen riesgos inherentes a la actividad minera (accidentes de trabajo y enfermedades profesionales propias de exposición a productos químicos en forma de humos, vapores y polvo en suspensión, entre otros), lo cual se refleja en el nivel de accidentes que ocurren, según su diversa intensidad".

En el periodo 1991 - 2017, en el sector minero peruano, ocurrieron 1,767 accidentes mortales y 39,993 accidentes incapacitantes y en Uchucchacua 37 accidentes mortales y 533 accidentes incapacitantes, que correspondieron a 246,601 días perdidos. (Ver Figuras 1 y 2).

Para conocer la tendencia de la accidentabilidad de la mina Uchucchacua, se contó con todos los Informes de Investigación periodo 2008-2017, en que se registraron 245 accidentes incapacitantes y 10 mortales con 68,727 días perdidos y un costo de US $\$ 2^{\prime} 153,282$.

\section{Número de Accidentes Incapacitantes}

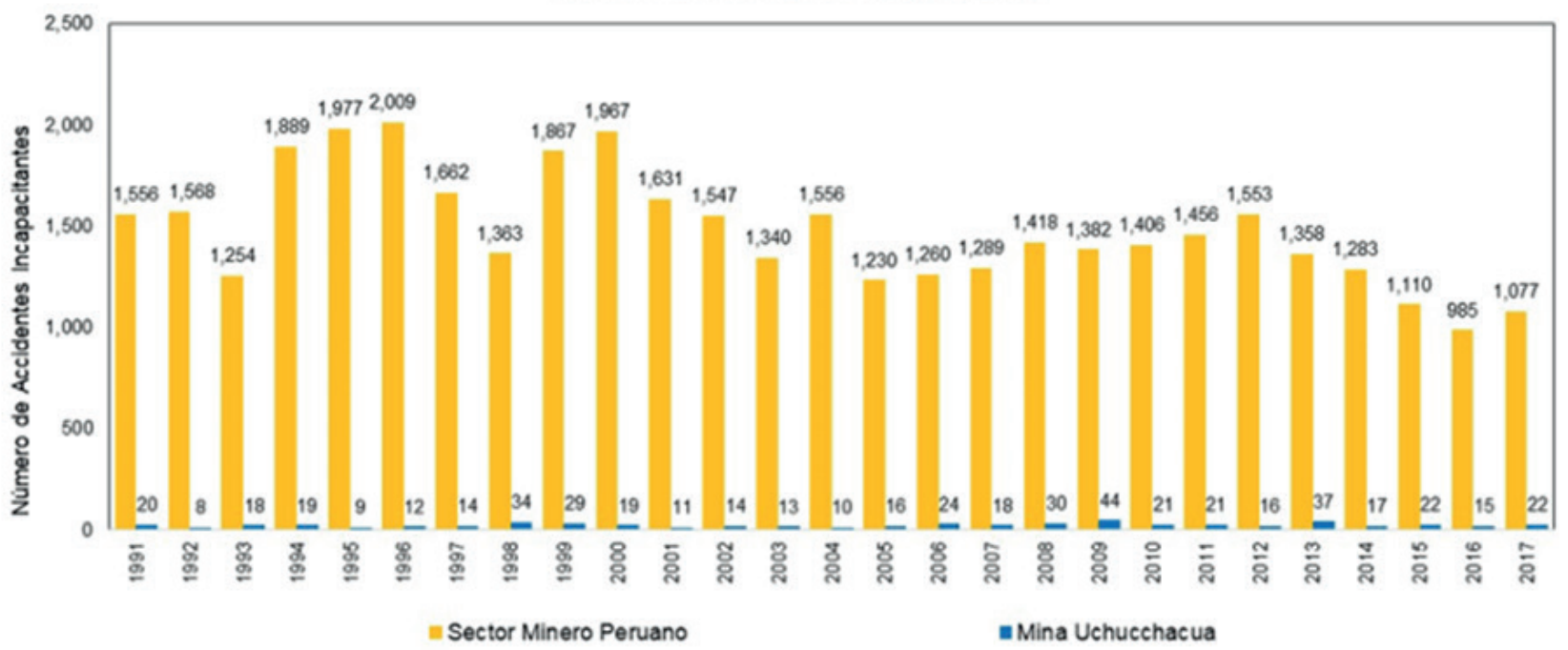

Figura 1. Accidentes incapacitantes: Minería Peruana - Uchucchacua: 1991 - 2017

Fuente: (Acero, T., s. f.)

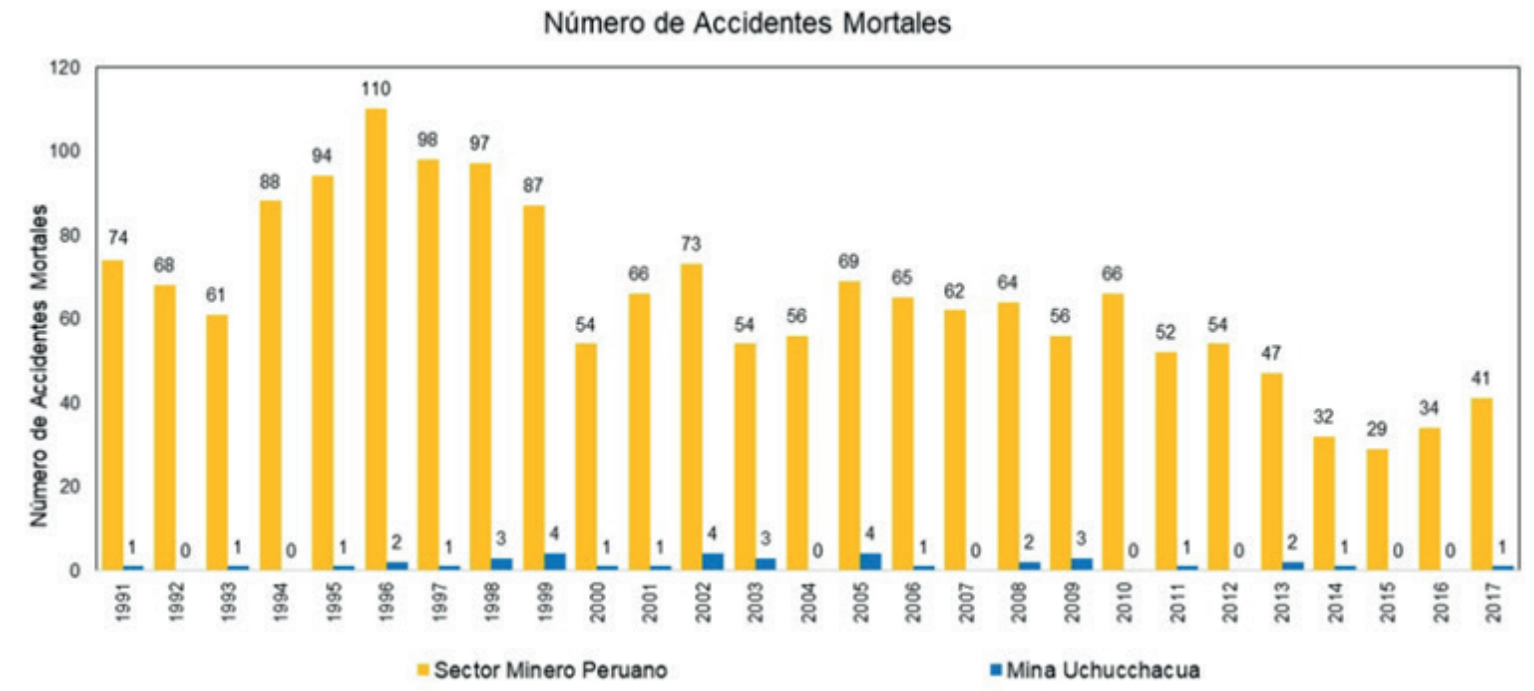

Figura 2. Accidentes mortales: Minería Peruana - Uchucchacua: 1991 - 2017

Fuente: Área de Seguridad de la Unidad Minera Uchucchacua (2018). 
En este periodo, los índices de Seguridad de la mina comparados con los del Sector Minero Nacional, superaron 7 años el índice de frecuencia y de severidad y en 6 años el índice de accidentabilidad (Figuras 3, 4 y 5). Los resultados señalados, ayudaron a decidir realizar esta investigación, con el fin de orientar las acciones necesarias para corregir esta tendencia.

La mina Uchucchacua está ubicada en la provincia de Oyón, Región de Lima. Es una mina subterránea de alta producción de plata. Presenta estructuras son potentes, emplazadas en calizas. Los métodos de explotación son el sublevel stoping y el corte y relleno ascendente. El procesamiento de mineral es por flotación, produciendo concentrados de plomo y plata.

(Juran et al., 2001) en el estudio de la calidad, destaca la importancia del (Diagrama de Pareto.pdf, s. f.), el cual resume de la siguiente manera: "cuando observé que los defectos de la calidad tienen unas frecuencias desiguales; esto es, cuando se ordenaba una larga lista de defectos según sus frecuencias, unos pocos de los defectos eran responsables de la mayor parte del problema". En el mismo sentido se pronuncian (Canavos, s. f.) y (Kume, 1992).

En esta lógica de Pareto, la información que se obtiene de los Informes de investigación de accidentes se usará el Principio de Pareto para realizar su evaluación y análisis. Se elaborarán estadísticas y diagramas, que ayudarán a reconocer los pocos parámetros que representan las cusas de la mayor cantidad de pérdidas. Así, se identificará también la tendencia de la accidentabilidad.

Por definición propia para esta investigación, público objetivo es el personal que resulta ser el más afectado por los accidentes. Sufre el daño del $20 \%$ de parámetros críticos, responsables del $80 \%$ de los accidentes. Es determinado mediante las estadísticas señaladas, y conocerlo es fundamental para involucrarlo en las actividades de prevención.

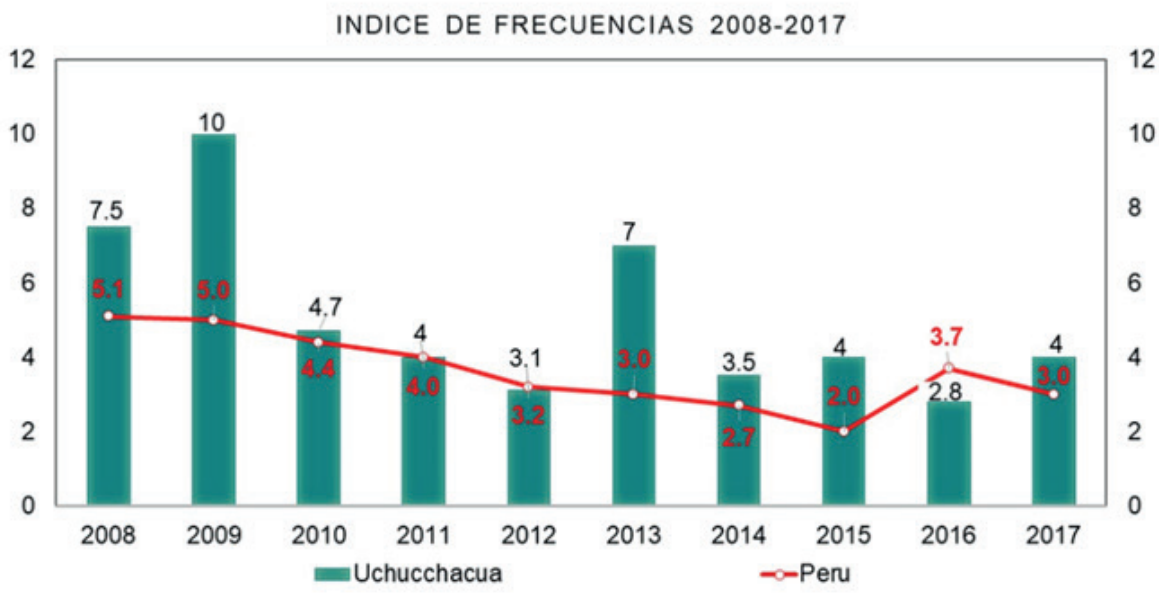

Figura 3. Índice de Frecuencias durante los años 2008 - 2017

Fuente: (Anuario Minero, 2018)

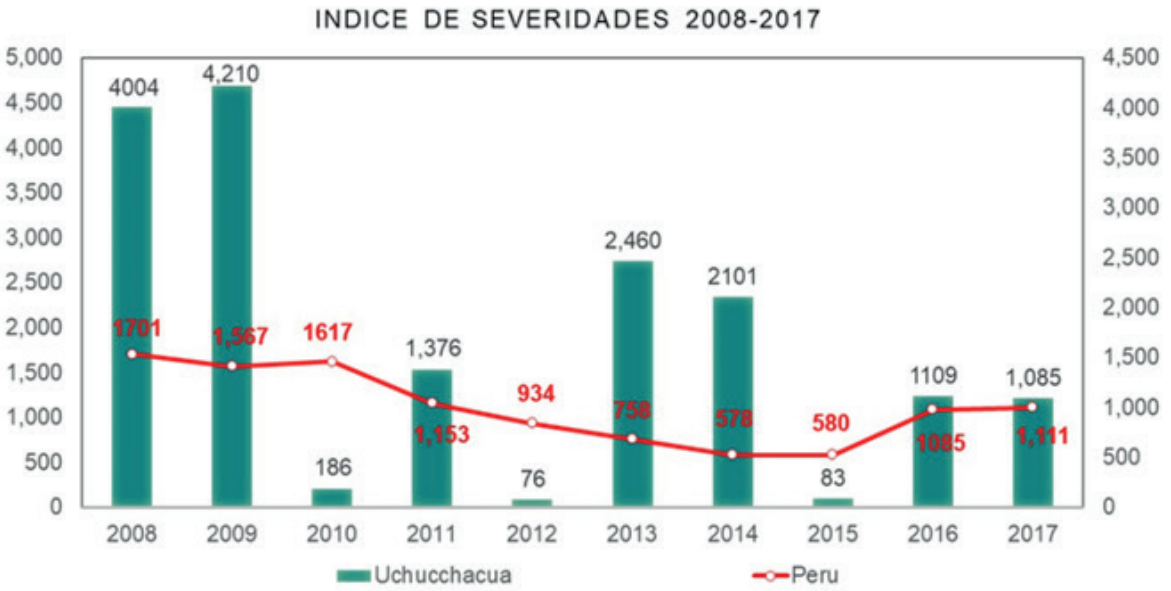

Figura 4. Índice de Severidad durante los años 2008 - 2017

Fuente: (Anuario Minero, 2018) 


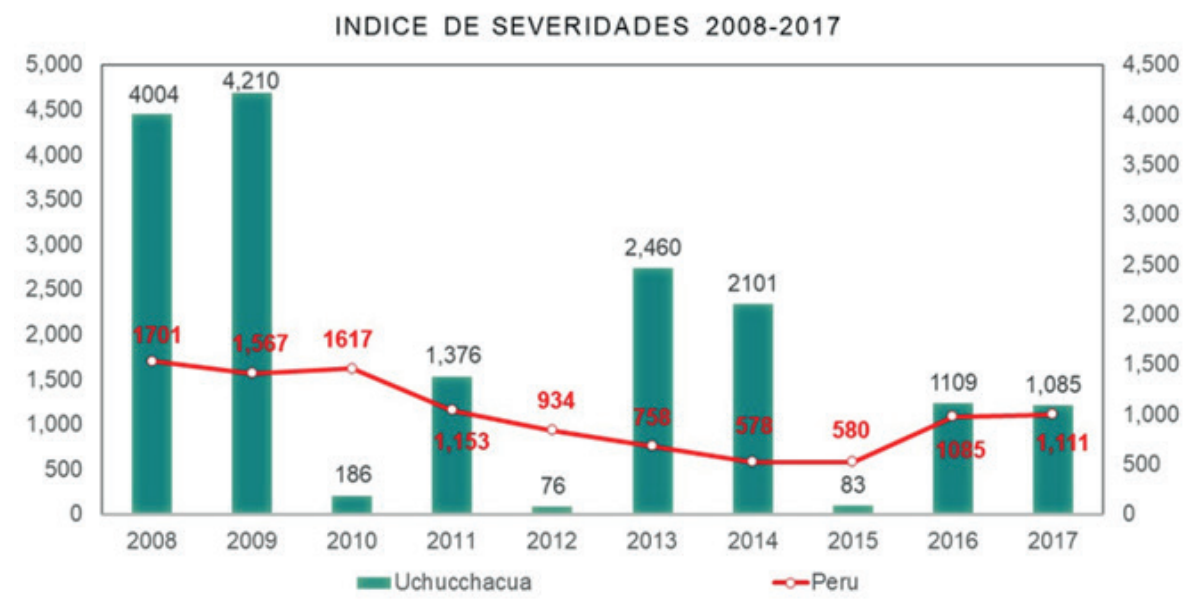

Figura 5. Índice de Accidentabilidad durante los años 2008 - 2017

Fuente: (Anuario Minero, 2018).

(Belmar, V., s. f.) considera que "la investigación de accidentes es una técnica preventiva orientada a detectar y controlar las causas que originan el accidente, con el fin de evitar la repetición de uno igual o similar. Cuando se investiga un accidente se debe llegar a establecer con la mayor precisión posible, cuáles fueron los actos y condiciones sub estándares que permitieron que el accidente ocurriera".

Otra herramienta utilizada en la investigación fue la TASC, técnica de investigación de accidentes (SCAT, por sus siglas en inglés) desarrollada por el International Loss Control Institute (ILCI), que "es un procedimiento que inicia con la evaluación potencial de perdida, definiendo su potencial de severidad de pérdida (mayor, grave o menor), la probabilidad de ocurrencia (alta, moderada y rara) y frecuencia de exposición (grande, moderada y baja)". Según refieren (Baylón Montes \& Santillán Gonzáles, 2019), esta herramienta fue propuesta por Bird y Germain, explicando la multicausalidad de los eventos (accidentes), identificando las causas directas y básicas.

\section{METODOS}

La información recopilada de accidentes mortales e incapacitantes, en la unidad minera objeto de este estudio, se registró en una base de datos, en Excel, En su evaluación y análisis se usaron tablas dinámicas, que son de gran versatilidad para gestionar datos.

Cada característica del trabajo analizada tuvo como marco la Tabla 1, con parámetros que la describen, establecidos conforme van apareciendo al revisar los Informes de Investigación y registrados, por una sola vez, en una hoja de excel. Paralelamente a cada parámetro se le asigna un código, que luego permitirá contabilizar su repetición. Las características del trabajo fueron clasificadas, según criterio del proponente, como: del ambiente de trabajo, del personal y de la organización del trabajo. Cada una de ellas fue evaluada y analizada, considerando su influencia en la tendencia de la accidentabilidad (Tabla 1).
Tabla 1. Características del trabajo de los accidentes evaluados

\begin{tabular}{|c|c|}
\hline $\begin{array}{c}\text { Característica del } \\
\text { trabajo }\end{array}$ & Descripción \\
\hline \multirow{3}{*}{$\begin{array}{l}\text { Evaluación de estadísticas } \\
\text { generales }\end{array}$} & - Tipo de riesgo o accidente \\
\hline & Público objetivo \\
\hline & - Clase de accidente \\
\hline \multirow{10}{*}{ Características del personal } & - Grado de instrucción \\
\hline & - Estado civil \\
\hline & - Parte del cuerpo afectado \\
\hline & Tipo de pérdida \\
\hline & Días perdidos \\
\hline & Experiencia total \\
\hline & - Experiencia en el puesto \\
\hline & Acto subestándar \\
\hline & - Factores personales \\
\hline & Sub factores personales \\
\hline \multirow{4}{*}{$\begin{array}{l}\text { Características del ambiente } \\
\text { de trabajo }\end{array}$} & - Departamento responsable \\
\hline & Área de trabajo \\
\hline & · Lugar específico \\
\hline & $\begin{array}{l}\text { Tipo de labor } \\
\text { Condiciones subestándar } \\
\text {. } \text { Factores de trabajo } \\
\text { Sub factores de trabajo }\end{array}$ \\
\hline \multirow{8}{*}{$\begin{array}{l}\text { Características de la } \\
\text { organización del trabajo }\end{array}$} & Rango de edad \\
\hline & - Hora \\
\hline & · Día \\
\hline & . Mes \\
\hline & · Año \\
\hline & - Costo \\
\hline & · Razón Social \\
\hline & - Turno de trabajo \\
\hline Medidas sistemáticas de control & - Medidas de control de los A \\
\hline
\end{tabular}

Fuente: Elaboración propia

En otra hoja de Excel, al anotar la información de los accidentes en cada característica del trabajo se registra el código que corresponde al parámetro que la describe, según lo asignado en la primera hoja, por ejemplo: para 
ocupación, para condiciones subestándares, etc. Luego, se genera Figura de Pareto que considera las veces que se repite un parámetro y muestra de manera descendente los porcentajes individuales, y el acumulado.

Los Figuras de Pareto, permiten determinar para cada característica evaluada los pocos parámetros críticos asociados que son lo que se ubican dentro del $80 \%$ del porcentaje acumulado. En esta investigación se consideró un porcentaje acumulado mayor, al incluir otros parámetros de igual porcentaje, aún ya se haya alcanzado el $80 \%$ acumulado. Con esta decisión se da igual importancia a parámetros que, sólo por ubicación en el Figura pudieran quedar excluidos. Así se determinan los pocos críticos y también se logra establecer, según corresponda, el público objetivo y sus características.

\section{RESULTADOS}

En este artículo se prioriza mostrar las tablas de los parámetros asociados a cada característica evaluada, que identifican los críticos, además porque los figuras presentan una estructura similar. Sólo se mostrarán las tablas estadísticas que corresponden a accidentes por caída de rocas, uno de los que requiere mayor control en minería.

\subsection{Evaluación y análisis de las características generales}

Las características generales permiten identificar los riesgos que se materializaron por tipo de accidente, ocupación y clase de accidente. Su identificación es un punto de partida importante porque orienta a cuáles riesgos debemos orientar nuestro control. Los resultados de la evaluación y análisis, son:

\subsubsection{Accidentes por tipo de riesgo o de accidente}

Se determinó que los pocos riesgos críticos que prevalecen en la accidentabilidad de la Unidad son 4. Estos generaron el $80.8 \%$ de los accidentes ocurridos: caída de rocas $(24.7 \%)$, manipuleo de materiales $(23.5 \%)$, operación de maquinarias y equipos $(19.2 \%)$ y caída de personas $(13.3$ $\%)$. Tabla 2 y Figura 6.

\subsubsection{Accidentes por público objetivo por caída de rocas}

Esta característica es una de las de mayor relevancia. Permite conocer los puestos de trabajo más afectados,

Tabla 2. Evaluación de los accidentes por tipo de riesgo

\begin{tabular}{|c|c|c|c|}
\hline Tipo de Accidente & Número & Porcentaje \% & Pareto (Acumulado) \\
\hline Caída de rocas & 63 & $24.7 \%$ & $24.7 \%$ \\
\hline Manipuleo de materiales & 60 & $23.5 \%$ & $48.2 \%$ \\
\hline Operación de maquinarias y equipos & 49 & $19.2 \%$ & $67.5 \%$ \\
\hline Caída persona & 34 & $13.3 \%$ & $80.8 \%$ \\
\hline Tránsito & 21 & $8.2 \%$ & $89.0 \%$ \\
\hline Acarreo y transporte & 14 & $5.5 \%$ & $94.5 \%$ \\
\hline Manipuleo de herramientas & 6 & $2.4 \%$ & $96.9 \%$ \\
\hline Energía Eléctrica & 4 & $1.6 \%$ & $98.4 \%$ \\
\hline Explosión / Intoxicación por gases & 4 & $1.6 \%$ & $100.0 \%$ \\
\hline Total general & 255 & $100.0 \%$ & \\
\hline
\end{tabular}

Fuente: Elaboración propia

TIPO DE ACCIDENTE

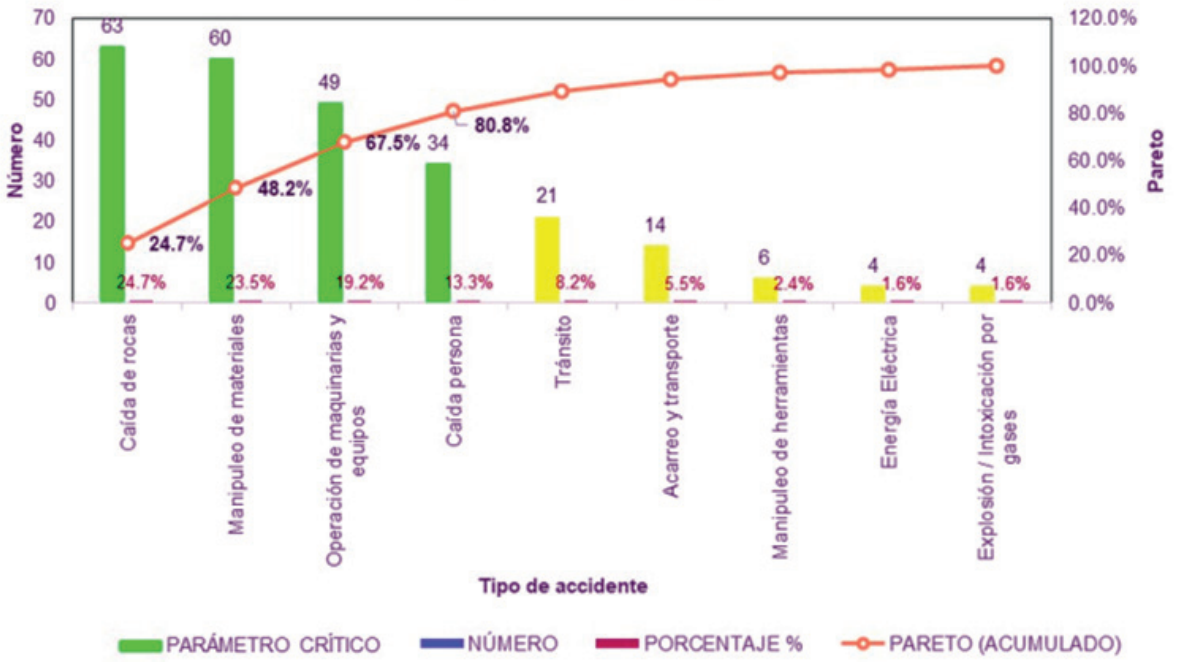

Figura 6. Identificación de los tipos de riesgos críticos que generan accidentes Fuente: Elaboración propia 
determinados al evaluar los accidentes por tipo de riesgo crítico. A este personal lo llamaremos público objetivo. Analizando otras características encontraremos más rasgos de este público, útiles para definir las medidas de control requeridas, incluyendo los que deben considerarse en los procesos de selección del personal. Conocidas las ocupaciones del público objetivo podremos disponer de nóminas de personal para involucrarnos en el diseño y asegurar su participación en las actividades preventivas

En este tipo de accidentes los puestos del público objetivo más afectados, son: perforista $(50.8 \%)$, ayudante de perforista $(28.6 \%)$ y operador de equipo pesado (7.9 $\%$ ), alcanzando el $87.3 \%$. (Tabla 3 ). El personal que realiza trabajos de perforación es el más afectado. Se tratan de puestos que realizan tareas en equipo y coordinadamente. El perforista opera el equipo, organiza y dirige las actividades en la labor.

\subsubsection{Accidentes según la experiencia en el puesto de trabajo por caída de rocas}

El personal accidentado por caída de rocas, según su experiencia en puesto fue: de 3 a 5 años $(27.0 \%)$ de 5 a 7 años $(17.5 \%)$, de 1 a 2 años $(15.9 \%)$, de 7 a 10 años (12.7\%), de 2 a 3 años $(9.5 \%)$ y menos de 1 año $(9.5 \%)$, totalizando el $92.1 \%$. En resumen, el personal que se accidenta tiene experiencia menor a 10 años, y el que tiene una experiencia menor a 5 años representa el $61.9 \%$. Esta información debe considerarse en los procesos de selección y formación del personal, su relevancia en la prevención de accidentes es importante (Tabla 4).

Relacionando el público objetivo con la de experiencia del personal en el puesto de trabajo, resulta que los ayudantes de perforistas, perforistas y operadores de equipo pesado, son los más afectados. El personal con una experiencia menor a 5 años está dentro del $63 \%$ de los accidentes. Este análisis debe orientar a la supervisión cuál debe ser la experiencia del personal para asignar el trabajo, asegurando que cuenten con las capacidades y habilidades requeridas, incluso para relacionarse con sus pares y con los diferentes niveles de la organización, y para lograr su predisposición a cumplir normas y prácticas de trabajo.

\subsubsection{Accidentes según los actos subestándares por caída de rocas}

Los actos subestándares críticos son: ubicación incorrecta $(52.5 \%)$ y omisión de asegurar $(28.8 \%)$ representando el $81.4 \%$ y que se relacionan con el grado de entrenamiento y actitud para cumplir estándares y prácticas. Las actitudes son permitidas y vistas como normales en el trabajo y no son corregidas. Es necesario motivar y mejorar la supervisión del personal (Tabla 5).

Tabla 3. Evaluación de estadísticas por público objetivo del personal accidentado por caída de rocas

\begin{tabular}{lccc}
\hline \multicolumn{1}{c}{ Público Objetivo } & Número & Porcentaje \% & Pareto (Acumulado) \\
\hline Perforista & 32 & $50.8 \%$ & $\mathbf{5 0 . 8 \%}$ \\
Ayudante Perforista & 18 & $28.6 \%$ & $\mathbf{7 9 . 4 \%}$ \\
Operador Equipo Pesado & 5 & $7.9 \%$ & $\mathbf{8 7 . 3 \%}$ \\
Ayudante Motorista & 2 & $3.2 \%$ & $90.5 \%$ \\
Supervisor & 2 & $3.2 \%$ & $93.7 \%$ \\
Capataz & 1 & $1.6 \%$ & $95.2 \%$ \\
Operador equipo shotcreate & 1 & $1.6 \%$ & $96.8 \%$ \\
Mecánico & 1 & $1.6 \%$ & $98.4 \%$ \\
Muestrero & 1 & $1.6 \%$ & $\mathbf{1 0 0 . 0 \%}$ \\
Total general & $\mathbf{6 3}$ & $\mathbf{1 0 0 . 0 \%}$ & \\
\hline
\end{tabular}

Fuente. Elaboración propia

Tabla 4. Evaluación de las estadísticas por experiencia en el puesto de trabajo por caída de rocas

\begin{tabular}{lccc}
\hline \multicolumn{1}{c}{ Experiencia en el Puesto } & Número & Porcentaje \% & Pareto (Acumulado) \\
\hline 3 a menos de 5 & 17 & $27.0 \%$ & $\mathbf{2 7 . 0 \%}$ \\
5 a menos de 7 & 11 & $17.5 \%$ & $\mathbf{4 4 . 4 \%}$ \\
1 a menos de 2 & 10 & $15.9 \%$ & $\mathbf{6 0 . 3} \%$ \\
7 a menos de 10 & 8 & $12.7 \%$ & $\mathbf{7 3 . 0 \%}$ \\
2 a menos de 3 & 6 & $9.5 \%$ & $\mathbf{8 2 . 5 \%}$ \\
menos de 1 año & 6 & $9.5 \%$ & $\mathbf{9 2 . 1} \%$ \\
10 a menos de 15 & 4 & $6.3 \%$ & $98.4 \%$ \\
20 a menos de 25 & 1 & $1.6 \%$ & $\mathbf{1 0 0 . 0 \%}$ \\
Total general & $\mathbf{6 3}$ & $\mathbf{1 0 0 . 0 \%}$ & \\
\hline
\end{tabular}


Al relacionar el público objetivo con actos subestándares, la omisión de asegurar prima en los motoristas, ayudantes de perforista y perforista; no desatar o hacerlo deficiente en perforistas y la ubicación incorrecta en ayudantes de perforista, operadores de equipo pesado y perforistas. Así, se reconoce al personal que debe ser prioritariamente entrenado y formado.

3.1.5 Accidentes según los factores personales por caída de rocas

Los factores personales que influyen en la ocurrencia de accidentes por caída de rocas, que acumulan el 93.2\% son: personal desmotivado $(67.8 \%)$, ahorro de tiempo y esfuerzo $(8.5 \%)$, falta de entrenamiento $(6.8 \%)$, falta de conocimiento $(5.1 \%)$ y falta de habilidad $(5.1 \%)$. La desmotivación tiene relación con la insatisfacción del colaborador, por: desinformación, limitada comunicación, no participa en la toma de decisiones, no reconocer sus logros, falta de facilidades, etc. Este comportamiento, responde al hecho que el personal no quiere hacer el trabajo adecuadamente, conjugado con la falta de capacidades y habilidades requeridas (Tabla 6).

Al correlacionar el público objetivo y factores personales resulta que los puestos más afectados son: el perforista por ahorro de tiempo y esfuerzo y desmotivado para la tarea; el ayudante de perforista por la falta de entrenamiento y la desmotivación para ejecutar la tarea; hay desmotivación en los ayudantes de motorista y operadores de equipo pesado. También hay falta de conocimiento y de habilidad, por lo que la capacitación y entrenamiento del personal en geomecánica, formas de sostenimiento de las labores incluyendo el entrenamiento para corregir su posición y ubicación al ejecutar el trabajo, es básica.

\subsubsection{Accidentes según el tipo de labor por caída de rocas}

Permite identificar en qué espacios específicos dentro de los lugares de trabajo y de las áreas se produjeron los accidentes. Son las labores donde el personal ejecuta su tarea. En cuatro labores específicas del Departamento de Minas se generan el $87.3 \%$ de los accidentes por caída de rocas. En el tajeo (labor de producción) el $50.8 \%$ y en las rampas, galerías y cruceros (labores de avance) el $36.5 \%$.

Los tajeos son las labores más críticas. El personal que labora en estas labores se constituye en público objetivo fundamental. Este debe ser convenientemente seleccionado y preparado para ejecutar las actividades planificadas. Las medidas preventivas de control a implementar son prioritarias para corregir las desviaciones que pudieran afectar su desempeño, así como garantizar la disponibilidad oportuna y uso eficiente de los recursos programados.

Las labores de avance se ejecutan hasta alcanzar su longitud de diseño. Se realizan siguiendo una secuencia de actividades como son: sostenimiento, perforación, voladura $\mathrm{y}$ uso de equipos para el retiro del material generado

Tabla 5. Evaluación de las estadísticas según los actos subestándares por caída de rocas

\begin{tabular}{lccc}
\hline \multicolumn{1}{c}{ Acto subestándar } & Número & Porcentaje $\%$ & Pareto (Acumulado) \\
\hline Ubicación incorrecta & 31 & $52,5 \%$ & $\mathbf{5 2 , 5 \%}$ \\
Omisión de asegurar & 17 & $28,8 \%$ & $\mathbf{8 1 , 4 \%}$ \\
No desatar / Desatado deficiente de labor & 5 & $8,5 \%$ & $89,8 \%$ \\
Posición inadecuada & 2 & $3,4 \%$ & $93,2 \%$ \\
Omitir sistemas de advertencia & 2 & $3,4 \%$ & $96,6 \%$ \\
Uso inadecuado / no uso de EPP & 1 & $1,7 \%$ & $98,3 \%$ \\
Maniobra inadecuada & 1 & $1,7 \%$ & $\mathbf{1 0 0 , 0} \%$ \\
Total general & $\mathbf{5 9}$ & $\mathbf{1 0 0 , 0 \%}$ & \\
\hline
\end{tabular}

Fuente. Elaboración propia

Tabla 6. Estadística de los Factores personales por la caída de rocas

\begin{tabular}{|c|c|c|c|}
\hline Factores de Trabajo & Número & Porcentaje \% & Pareto (Acumulado) \\
\hline Personal desmotivado para la tarea & 40 & $67,8 \%$ & $67,8 \%$ \\
\hline Ahorro de tiempo y esfuerzo & 5 & $8,5 \%$ & $76,3 \%$ \\
\hline Falta de entrenamiento & 4 & $6,8 \%$ & $83,1 \%$ \\
\hline Falta de conocimiento & 3 & $5,1 \%$ & $88,1 \%$ \\
\hline Falta de habilidad & 3 & $5,1 \%$ & $93,2 \%$ \\
\hline No respetar prácticas /estándares establecidos & 2 & $3,4 \%$ & $96,6 \%$ \\
\hline Desatención a los sistemas de reporte de advertencia & 1 & $1,7 \%$ & $98,3 \%$ \\
\hline Inadecuado discernimiento, no elige opción correcta & 1 & $1,7 \%$ & $100,0 \%$ \\
\hline Total general & 59 & $100,0 \%$ & \\
\hline
\end{tabular}

Fuente. Elaboración propia 
producto de los disparos. Al realizar estas actividades ocurren los eventos. En todos los casos, la selección y formación del personal es fundamental (Tabla 7).

Al relacionar el público objetivo con tipo de labor en accidentes por caída de rocas, los perforistas se han accidentado principalmente en tajeos, rampas, galerías, cruceros y chimeneas convencionales y los ayudantes de perforista en galerías, rampas y tajeos.

Al relacionar los actos subestándares con el tipo de labor para los accidentes por caída de rocas, se concluye que: el no desatar o realizar el desatado deficiente de la labor es notorio en rampas y tajeos; la omisión de asegurar en las chimeneas convencionales, cruceros, rampas y tajeos; omitir sistemas de advertencia en tajeos; ubicación incorrecta del personal para el desatado en galerías, rampas $\mathrm{y}$ tajeos.

$\mathrm{Al}$ relacionar los factores personales con el tipo de labor, los que más influyen son: ahorro de tiempo y esfuerzo, falta de conocimiento del personal, falta de entrenamiento y no respetar prácticas y estándares establecidos en los tajeos y personal desmotivado para las tareas en todos los tipos de labor evaluados. Esta situación es grave y tiene que ver con la predisposición espontánea del personal para cumplir las medidas preventivas que se adopten. Adicionalmente la falta de conocimiento y entrenamiento, denota que al personal carece de capacidades para ejecutar su trabajo, situación que influye en los resultados y en el relacionamiento con la supervisión y pares.

El comportamiento del personal anteriormente descrito puede identificarse mediante observaciones de trabajo. Esta medida de control debe ser implementada ampliamente como parte del sistema de Gestión de la Unidad. También será importante establecer un adecuado plan para reforzar el entrenamiento del personal, así como las capacidades del personal para aplicar conocimientos en geomecánica para determinar los trabajos de sostenimiento de las labores, el cual debe hacerse siguiendo un estándar y en la oportunidad debida.

La planificación oportuna de todas las actividades es fundamental. Sin éste elemento de la Administración, el control de los trabajos será imposible, pues al no contar con un diseño o ingeniería previa, por ejemplo, no se podrán conocer los parámetros que deben evaluarse en campo, ni los estándares y prácticas a seguir, entre otros

\subsubsection{Accidentes según las condiciones subestándar por caída de rocas}

Las condiciones subestándares permiten determinar los peligros que no fueron identificados previo al evento. Estos favorecen la materialización de los riesgos, por lo que su identificación es necesaria y su atención debe ser oportuna y sistemática.

En los accidentes por caída de rocas, cuatro son las condiciones subestándares predominantes que representan el $82.5 \%$ : labor inestable (47.6\%), labor inadecuadamente sostenida y/o no se descarga el material suelto de las labores $(17.5 \%)$, falta o inadecuada barrera, guarda o berma de seguridad $(11.1 \%)$ y falta o deficiencia de equipos, herramientas o materiales $(6.3 \%)$. Es necesario implementar adecuadas prácticas, procedimientos, estándares, habilidades y capacidades en el personal y ejecutar acciones oportunas de seguimiento y control. Paralelo a ello, debe garantizarse el suministro y disponibilidad de recursos con la calidad y en la oportunidad requerida (Tabla 8).

Al relacionar el público objetivo con condiciones subestándar, resulta que las principales causas de los accidentes de los perforista, son: caminos, pisos o superficies inadecuadas o con desperdicios, carga suspendida, falta de herramientas o materiales, inadecuadas barreras o guardas de seguridad, labor inadecuadamente desatada o sostenida y principalmente por exponerse a labores inestables; los ayudantes de perforistas, por trabajar en labores inadecuadamente desatada o sostenidas y por hacerlo en labores inestables y el operador de equipo pesado por acceder a labores inestables. Este tipo de exposiciones del personal tienen que ver con aspectos conductuales o acceder sumisamente a exigencias para ejecutar el trabajo. Una adecuada supervisión es fundamental para corregir estos resultados.

Al relacionar condición subestándar versus tipo de labor se encontró que hay existencia de espacios de trabajo reducidos para realizar la tarea, por diseño o congestión

Tabla 7. Evaluación de las estadísticas de accidentes según el tipo de labor critico por caída de rocas

\begin{tabular}{|c|c|c|c|}
\hline Tipo de Labor & Número & Porcentaje \% & Pareto (Acumulado) \\
\hline Tajeo & 32 & $50.8 \%$ & $50.8 \%$ \\
\hline Rampa & 11 & $17.5 \%$ & $68.3 \%$ \\
\hline Galería & 7 & $11.1 \%$ & $79.4 \%$ \\
\hline Crucero & 5 & $7.9 \%$ & $87.3 \%$ \\
\hline Chimenea convencional & 3 & $4.8 \%$ & $92.1 \%$ \\
\hline Subnivel & 3 & $4.8 \%$ & $96.8 \%$ \\
\hline Tolva minera & 1 & $1.6 \%$ & $98.4 \%$ \\
\hline Chimenea RC & 1 & $1.6 \%$ & $100.0 \%$ \\
\hline Total general & 63 & $100.0 \%$ & \\
\hline
\end{tabular}

Fuente. Elaboración propia 
de materiales; falta de equipamiento, herramientas o de materiales; falta de barreras, guardas o los que existen están en condiciones inadecuadas en las galerias y tajeos; labores inadecuadamente desatadas o sostenidas en galerias, rampas, tajeos y labores inestables en cruceros, rampas, subniveles y tajeos.

Estas condiciones subestándar existen en las labores de producción de mineral y en las principales labores de acceso. Estos peligros pueden ser identificados con un adecuado sistema de inspecciones a realizar por la supervisión. El personal también tiene responsabilidad en aceptar realizar trabajos manteniendo las deficiencias señaladas. Esta información permitirá priorizar acciones especificas.

\subsubsection{Accidentes según los factores de trabajo por caída de rocas}

Los factores de trabajo explican el porqué de la existencia de condiciones subestándar. Dependen básicamente de la supervisión y de la dirección de la organización. En el caso de accidentes por caída de rocas, tres son los factores de trabajo que requieren toda la atención: procedimientos y prácticas inadecuadas $(55.6 \%)$, estándares inadecuados $(20.6 \%)$ y liderazgo y/o supervisión deficiente (15.9\%). Este resultado se explica porque el personal accidentado no ha sido adecuadamente informado, entrenado, capacitado para ejecutar su trabajo y no dispone de los recursos definidos en los estándares para cumplir con su trabajo. La supervisión requerirá mejorar considerablemente su desempeño para contribuir eficazmente al control de los factores señalados (Belmar, V., s. f.) (Tabla 9).

Al relacionar los Factores de trabajo con el público objetivo, se determina que los ayudantes de perforistas han estado expuestos por seguir estándares inadecuados, liderazgo y/o supervisión deficiente y adoptar procedimientos y prácticas inadecuadas; los operadores de equipo pesado por seguir procedimientos y prácticas inadecuadas y los perforistas no cumplir adecuados estándares, seguir a un liderazgo y/o supervisión deficiente y no cumplir procedimientos y prácticas adecuadas para el trabajo. Las acciones concretas a desarrollarse para corregir estas causas básicas son de responsabilidad de la dirección que es la encargada de planificar, organizar, liderar y sobretodo controlar el trabajo.

Al relacionar los Factores de trabajo con el tipo de labor se determinó que: se siguen estándares inadecuados en rampas y tajeos; el liderazgo y/o supervisión deficiente es notoria en galerias, rampas y tajeos y el seguir procedimeintos y prácticas inadecuadas en cruceros, galerias, rampas y tajeos. La efectiva atención de estos factores de trabajo implica la participación activa del personal en la definición de los estándares, procedimientos y prácticas existentes y en su difución. También debe

Tabla 8. Evaluación de las estadísticas de condiciones subestándar que generan los accidentes por caída de rocas

\begin{tabular}{lccc}
\hline Condición subestándar & Número & Porcentaje $\%$ & Pareto (Acumulado) \\
\hline Labor inestable & 30 & $47.6 \%$ & $\mathbf{4 7 . 6 \%}$ \\
Labor inadecuadamente desatada / sostenida / no se descarga material suelto & 11 & $17.5 \%$ & $\mathbf{6 5 . 1 \%}$ \\
Falta o inadecuada barrera, guarda, bermas, etc. de Seguridad & 7 & $11.1 \%$ & $\mathbf{7 6 . 2} \%$ \\
Falta de equipamiento, herramienta o materiales o deficiencia & 4 & $6.3 \%$ & $\mathbf{8 2 . 5 \%}$ \\
Caminos, pisos, superficies inadecuadas o con desperdicios & 3 & $4.8 \%$ & $87.3 \%$ \\
Espacio reducido para la tarea / congestión & 2 & $3.2 \%$ & $90.5 \%$ \\
Carga suspendida & 2 & $3.2 \%$ & $93.7 \%$ \\
Herramientas, equipos defectuosos/ inadecuados/ sin mantenimiento & 1 & $1.6 \%$ & $95.2 \%$ \\
Sobre excavación & 1 & $1.6 \%$ & $96.8 \%$ \\
Falta de orden y limpieza & 1 & $1.6 \%$ & $98.4 \%$ \\
Falta de plataforma/ o inadecuada & 1 & $1.6 \%$ & $\mathbf{1 0 0 . 0 \%}$ \\
Total general & $\mathbf{6 3}$ & $\mathbf{1 0 0 . 0 \%}$ & \\
\hline
\end{tabular}

Fuente. Elaboración propia

Tabla 9. Evaluación de la estadística de Factores de trabajo que generan los accidentes por caída de rocas

\begin{tabular}{lccc}
\hline \multicolumn{1}{c}{ Factores de Trabajo } & Número & Porcentaje \% & Pareto (Acumulado) \\
\hline Procedimientos, prácticas inadecuadas & 35 & $55.6 \%$ & $\mathbf{5 5 . 6 \%}$ \\
Estándares inadecuados & 13 & $20.6 \%$ & $\mathbf{7 6 . 2 \%}$ \\
Liderazgo y/o Supervisión deficiente & 10 & $15.9 \%$ & $\mathbf{9 2 . 1 \%}$ \\
Ingeniería inadecuada & 2 & $3.2 \%$ & $95.2 \%$ \\
Disciplina inadecuada & 1 & $1.6 \%$ & $96.8 \%$ \\
Evaluación deficiente de exposición a pérdidas & 1 & $1.6 \%$ & $98.4 \%$ \\
Falta de procedimientos, prácticas & 1 & $1.6 \%$ & $\mathbf{1 0 0 . 0 \%}$ \\
Total general & $\mathbf{6 3}$ & $\mathbf{1 0 0 . 0 \%}$ & \\
\hline
\end{tabular}

Fuente. Elaboración propia 
aseguarse el provisionamiento de los materiales, herramientas y equipos en adecuadas condiciones para el trabajo.

La supervisión requiere ser adecuadamente seleccionada y mejorar su desempeño, através del desarrollo de actividades técnicas, impulsar sus capacidades administrativas y reforzar sus habilidades blandas, para su intervención en los procesos y sobretodo sea el ejemplo, para el personal, con el fin de que su liderazgo ejerza todos sus efectos.

\section{DISCUSIÓN}

\subsection{Medidas sistemáticas de control}

Las medidas de control que se establecen corresponden a las que han sido registradas en los informes de investigación, pero que a la vez han sido revisadas, como parte de esta investigación, teniendo como base la Técnica de Análisis Sistemático de Causas. Su identificación permite establecer actividades que atenderán las características del trabajo evaluadas y analizadas, para evitar la ocurrencia de accidentes.

Siguiendo el Principio de Pareto podemos señalar que las medidas prioritarias de control, para el $81.7 \%$ de los accidentes reportados, son:

- Informar al personal de los accidentes ocurridos (11.9\%), el propósito es generar conciencia de los hechos y buscar principalmente mejorar las conductas y decisiones que se tomen

- Mejorar el control y sensibilización de la supervisión $(10.3 \%)$, este es un aspecto fundamental, la supervisión tiene que ser el ejemplo que debe seguir la fuerza laboral y la que se encarga de cristalizar en las labores la planificación, organización, dirección y control de las mismas;

- Retroalimentar al personal para seguir estándares, prácticas y procedimientos, buscando que internalicen la filosofía de estos elementos del Sistema de Gestión que permiten definir el qué y cómo hacer adecuadamente el trabajo

- Implementar e informar las observaciones de trabajo, este elemento es fundamentalmente conductual y permite corregir decisiones, actitudes y aspectos motivacionales del porqué la persona tiene un determinado desempeño

- Revisar y difundir los estándares de inspecciones y difundir resultados y corregir observaciones. Las inspecciones permiten identificar los peligros que son la base de la gestión de los riesgos, por ello que éste elemento de gestión debe ser adecuadamente implementado

- Definir estándar para retroalimentar, capacitar y entrenar permanentemente al personal. Es fundamental porque se orienta a la formación del personal, aspecto importante para un adecuado desenvolvimiento que beneficia la gestión de los riesgos y la productividad en la empresa

- Controlar los diseños de ingeniería. Muchos de los accidentes han ocurrido porque se han ejecutado trabajos en áreas inadecuadamente diseñadas, que no han tenido control previo. Es importante darle toda la atención necesaria a esta variable.

- Estandarizar la inducción y retroalimentación específica para el puesto. Debe realizarse tomando en cuenta todos los parámetros que lo afectan.

- Mecanizar la ejecución de la tarea, con el fin de evitar el usos de equipos inadecuados, posturas o realizar sobreesfuerzos que afecta la salud del personal.

- Hacer seguimiento a los planes de mantenimiento y corregir deficiencias, cuyas ventajas de por si son concluyentes tanto para garantizar que el personal opere un equipo en buenas condiciones y para obtener los resultados esperados, evitando incluso factores psicosociales que pueden afectar aún más a la fuerza laboral

- Revisar el IPERC. Esto se debe realizar de manera continua y motivando la participación del personal incluso para situaciones de cambio.

- Implementar sistemas de control para el manipuleo de materiales y accesorios, principalmente para garantizar el uso adecuado de recursos previstos y dirigir y asegurar la ejecución de maniobras.

\section{CONCLUSIONES}

- La información que proporciona los Informes de Investigación de los accidentes es certera y valiosa. Ésta adecuadamente evaluada y analizada, identifica las características del trabajo que influyen en la ocurrencia de los accidentes y permite determinar el público objetivo, que resulta ser el más afectado.

- Las medidas de control a implementar y de manera sistemática y preventiva, responden a corregir las tendencias actuales de la accidentabilidad, atendiendo los principales riesgos y parámetros críticos de las características reportadas.

- Los principales tipos de riesgo en la mina Uchucchacua son: caída de rocas, manipuleo de materiales, operación de maquinarias y equipos, y caída de personas. Estos inciden en el 19.2\% de las causas de los 206 accidentes (80.8\%), comprobándose que la aplicación del Principio de Pareto, cumple con lo establecido, ya que, para el amplio número de accidentes evaluados, aproximadamente el $\mathbf{8 0} \%$ de las consecuencias proviene del $\mathbf{2 0} \%$ de las causas.

- Las principales ocupaciones de los accidentados pertenecen al departamento de Minas (perforista, ayudante de perforista, operadores de quipos y 
maquinarias y Supervisor), Mantenimiento general (mecánico y soldador) y Planta de procesos (operador de planta). Así, los accidentes ocurren mayoritariamente en los departamentos operativos donde existe mayor exposición del personal a los riesgos, durante la ejecución de las actividades y tareas.

- Los resultados obtenidos producto de esta investigación permitirán orientar y utilizar adecuadamente los recursos necesarios para implementar las actividades propuestas. Dado el carácter específico deben estar orientadas al personal objetivo que podremos identificar bajo "listado" para que participen activamente y se comprometan con el cumplimiento de las medidas que se adopten.

- La Supervisión y Alta Dirección juegan un papel importante en la aplicación de los resultados de esta evaluación y análisis de los accidentes. Ambos son sinónimos de ejemplo, con el cual el personal guiará su accionar. Es fundamental que este nivel de la administración esté comprometido con los cambios propuestos y cumpla a cabalidad sus funciones.

- Esta investigación podrá ser útil para otras propuestas, puede ser una guía para orientar nuevas investigaciones, siempre reforzando la iniciativa de contar con elementos viables pero certeros para priorizar la prevención en nuestras operaciones.

- El desarrollo de esta investigación alcanzó sus objetivos: ser una herramienta analítica que permite evaluar los riesgos críticos en cada Departamento y finalmente tipo de labor en toda la Unidad Uchucchacua. de la mina Uchucchacua, y permitir promocionar la prevención basado en un enfoque de mayor jerarquía en el control de riesgos en el orden: eliminación, sustitución, controles de ingeniería, señalización advertencias y controles administrativos, y equipo de protección personal.

\section{AGRADECIMIENTOS}

El agradecimiento al Instituto de Investigación de la Facultad de Geología, Minas, Metalurgia y Ciencias Geográfica de la Universidad Nacional Mayor de San Marcos, asimismo expresar la gratitud a los colegas sanmarquinos por su preocupación por la ciencia y la investigación.

\section{REFERENCIAS}

Acero, T. (s. f.). Recuperado 8 de junio de 2020, de http://cybertesis. unmsm.edu.pe/bitstream/handle/20.500.12672/1830/ Acero_rt.pdf? sequence $=1$

Anuario Minero. (2018). Anuario Minero MINEM. Ministerio de Energia y Minas-MINEM, 114

Baylón Montes, J. C., \& Santillán Gonzáles, P. M. (2019). Determinantes de los accidentes en proyectos de gran envergadura en el sector construcción. Universidad ESAN. https://repositorio.esan.edu.pe///handle/20.500.12640/1600

Belmar, V. (s. f.). Recuperado 8 de junio de 2020, de https://www. isem.org.pe/portal/files/recurso/revista/90.pdf

Canavos, G. (s. f.). PROBABILIDAD Y ESTADÍSTICA. Aplicaciones ymétodos. George C. Canavos.pdf. Recuperado 8 de junio de 2020, de https://www.academia.edu/31905164/ PROBABILIDAD_Y_ESTAD\%C3\%8DSTICA. Aplicaciones_y_m\% $\overline{\mathrm{C}} 3 \% \overline{\mathrm{A}} \mathrm{A} 9$ todos._George_C._Canavos. pdf

Decreto Supremo D.S. N 024-2016-EM. (2016). Diario Oficial El Peruano, Lima, Perú, 56.

Diagrama de Pareto.pdf. (s. f.). Recuperado 8 de junio de 2020, de https://cursa.ihmc.us/rid=1KHL10SPR-1S0VNB2$18 \mathrm{MG} /$ pareto.pdf

Guzmán, L. (s. f.). Recuperado 8 de junio de 2020, de http://www.sigweb.cl/wp-content/uploads/biblioteca/ SeguridadLaboralChile.pdf

Juran, J. M., Godfrey, A. B., Hoogstoel, R. E., \& Schilling, E. G. (2001). Manual de calidad de juran (Quinta edición). McGraw-Hill.

Kume, H. (1992). Herramientas estadísticas básicas para el mejoramiento de la calidad. Editorial Norma. https:// books.google.com.pe/books?id=x4PnjSZYzMEC\&printsec $=$ frontcover $\mathrm{H} \mathrm{v}=$ onepage $\& \mathrm{q} \& \mathrm{f}=$ false

Van Neck, J. (2012, marzo 19). BRIE, a tool to detect and assess risks in small and medium sized enterprises. 30th International Congress on Occupational Health (March 1823, 2012). http://icoh.confex.com/icoh/2012/webprogram/ Paper7867.html

Velásquez Palacios, J., Chircca Ayesta, G., Alejo Contreras, P., \& Zamora Díaz, P. (2016). Análisis de los factores que afectan la accidentabilidad minera con énfasis en los sistemas de trabajo. Escuela de Postgrado GËRENS. http://repositorio. gerens.edu.pe/handle/Gerens/20 
